\title{
Acid-Induced Pain and Its Modulation in Humans
}

\author{
Nicholas G. Jones, ${ }^{1}$ Rebeccah Slater, ${ }^{1}$ Herve Cadiou, ${ }^{2}$ Peter McNaughton, ${ }^{2}$ and Stephen B. McMahon ${ }^{1}$ \\ ${ }^{1}$ Sensory Function Group, Center for Neuroscience, King's College London, London, SE1 1UL, United Kingdom, and ${ }^{2}$ Department of Pharmacology, \\ University of Cambridge, Cambridge, CB2 1PD, United Kingdom
}

\begin{abstract}
Despite the discovery of ion channels that are activated by protons, we still know relatively little about the signaling of acid pain. We used a novel technique, iontophoresis of protons, to investigate acid-induced pain in human volunteers. We found that transdermal iontophoresis of protons consistently caused moderate pain that was dose-dependent. A marked desensitization occurred with persistent stimulation, with a time constant of $\sim 3 \mathrm{~min}$. Recovery from desensitization occurred slowly, over many hours. Acid-induced pain was significantly augmented in skin sensitized by acute topical application of capsaicin. However, skin desensitized by repeated capsaicin application showed no significant reduction in acid-induced pain, suggesting that both capsaicin-sensitive and insensitive sensory neurons contribute to acid pain. Furthermore, topical application of non-steroidal anti-inflammatory drugs (NSAIDs) significantly attenuated acid-evoked pain but did not affect the heat pain threshold, suggesting a specific interaction between NSAIDs and peripheral acid sensors. Subcutaneous injection of amiloride $(1 \mathrm{~mm})$ also significantly inhibited the pain induced by iontophoresis of acid, suggesting an involvement of acid-sensing ion channel (ASIC) receptors. Conversely, iontophoresis of acid over a wide range of skin temperatures from 4 to $40^{\circ} \mathrm{C}$ produced only minor changes in the induced pain. Together these data suggest a prominent role for ASIC channels and only a minor role for transient receptor potential vanilloid receptor-1 as mediators of cutaneous acid-induced pain.
\end{abstract}

Key words: pain; acid; iontophoresis; ASIC; TRPV1; NSAID

\section{Introduction}

Acid causes pain, a fact that most people are all too aware of through accidental contact with exogenous acid. Acid pain is also of clinical relevance because tissue acidosis occurs in various physiological and pathophysiological states, including arthritis, ischemia, inflammation, and cancer, and this acidosis is thought to contribute to the pain experienced in these conditions (Issberner et al., 1996; Pan et al., 1999; Stubbs et al., 2000; Sutherland et al., 2001; Garber, 2003). Although it has been known for some time that protons excite nociceptors via the activation of inward cation-selective currents (Krishtal and Pidoplichko, 1981), the molecular identity of the ion channel or channels responsible for signaling acid $\mathrm{pH}$ has remained uncertain. Only recently have several candidate genes been cloned that code for ion channels that are activated by acid. These are the transient receptor potential vanilloid receptor-1 (TRPV1) (Caterina et al., 1997; Tominaga et al., 1998) and the acid-sensing ion channel (ASIC) family (Waldmann et al., 1997a) of which four have been cloned to date (ASICs 1-4) (Price et al., 1996; Lingueglia et al., 1997; Waldmann et al., 1997a,b; Chen et al., 1998; Gründer et al., 2000). The ability of the ASICs to assemble as functional heteromeric channel complexes as well as homomeric channels (the kinetics and $\mathrm{pH}$ sensitivity of which are determined by the specific subunit combinations) (Hesselager et al., 2004) further increases the number of

Received July 1, 2004; revised Aug. 27, 2004; accepted Sept. 30, 2004

This work was supported by the Biotechnology and Biological Sciences Research Council (N.G.J., H.C., P.M., and S.B.M.) and the Wellcome Trust (R.S. and S.B.M.).

Correspondence should be addressed to Dr. Nicholas Jones, Center for Neuroscience, Hodgkin Building, King's College London, Guy's Campus, London Bridge, SE1 1UL, UK. E-mail: Nicholas.2.jones@kcl.ac.uk.

D01:10.1523/JNEUROSCI.2619-04.2004

Copyright $\odot 2004$ Society for Neuroscience ～0270-6474/04/2410974-06\$15.00/0 candidate acid sensors. This notion that heteromeric ion channels exist and contribute to the native acid-evoked current and acid-induced pain is supported by the observation that neither the TRPV1 (Tominaga et al., 1998) nor homomultimeric ASICs (Hesselager et al., 2004) generate currents that have identical properties to native acid-evoked currents, which typically consist of both transient and sustained components, recorded from dorsal root ganglion (DRG) neurons (Xie et al., 2002). In fact, only ASIC 2a and ASIC 3 expressed homologously or heterologously demonstrate both of these current characteristics (Hesselager et al., 2004). It is known, however, that DRG neurons do express both TRPV1 (Caterina et al., 1997) and the majority of the ASIC subunits (Olson et al., 1998; Alvarez de la Rosa et al., 2002; Kellenberger and Schild, 2002).

In an attempt to identify some of the mechanisms responsible for signaling acid-induced pain in man, we have undertaken experiments on normal human volunteers. Previous experiments on human volunteers have already investigated aspects of acid pain (Steen and Reeh, 1993; Steen et al., 1995a,b; Issberner et al., 1996; Ugawa et al., 2002). However, the delivery methods used were invasive and caused pain themselves. Therefore, we have used a novel noninvasive method, transcutaneous iontophoresis, to deliver protons in a controlled manner, which itself does not cause pain, to characterize the features of acid-induced pain.

\section{Materials and Methods}

Subjects. Experiments were performed on healthy volunteers (male and female; $n=33$ ) aged between 21 and 47. In accordance with the Declaration of Helsinki, written consent was given by the subjects before participation in the study. Exclusions included pregnancy, asthma, any chronic skin condition, or known sensitivity to nonsteroidal anti- 

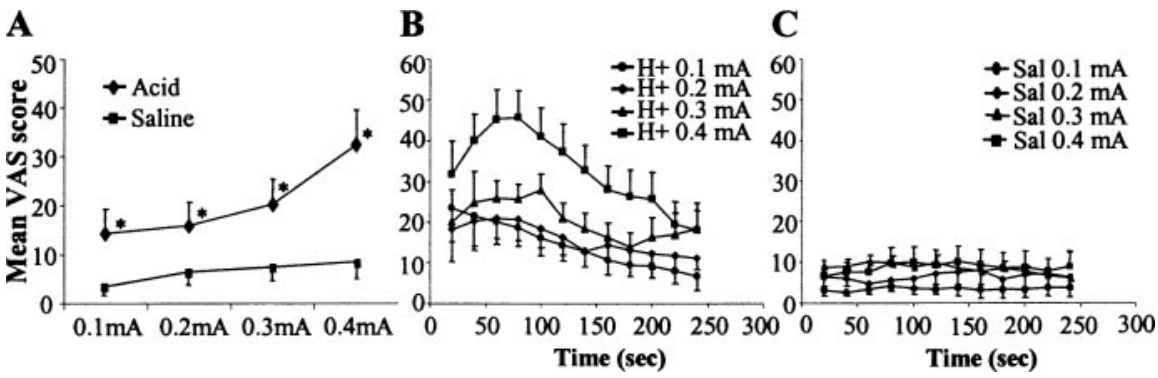

Figure 1. Mean pain rating and time course of perceived pain during iontophoresis of acid and saline. $A$, The mean VAS scores increase with the increase in current applied to the cathode, indicating that the perceived pain increases in a dose-dependent manner. Significantly higher VAS scores were obtained when acid was iontophoresed when compared with iontophoresis of saline. $B$, The time course of the mean VAS scores obtained when acid is iontophoresed at different currents $(0.1-0.4 \mathrm{~mA})$. $C$, The time course of the mean VAS scores obtained when saline was iontophoresed at different currents $(0.1-0.4 \mathrm{~mA})(n=12)$. Asterisk denotes a significant difference $(p<0.05)$.

inflammatory drugs (NSAIDs). The subjects were blind to all treatments and test solutions applied. Subjects underwent an initial training session to familiarize them with the testing and rating procedures and then attended one or more sessions, each lasting $\sim 1-2 \mathrm{hr}$. All procedures and protocols were approved by St. Thomas' Hospital Ethics Committee.

Iontophoresis of test solutions. Skin on the volar forearm of healthy adult volunteers was used in this study. After the experimental skin was cleaned with an alcohol swab, an iontophoresis chamber was attached with an adhesive disc, and an indifferent electrode was placed at a distant site usually on the hand. The chamber, $9 \mathrm{~mm}$ in diameter, was filled with acid (hydrochloric acid, $\mathrm{pH} 2$ ). Constant currents (0.1-0.4 mA) were then applied for $4 \mathrm{~min}$ to the cathode, located in the iontophoresis chamber, with a commercial iontophoresis unit (Phoresor II PM 700; IOMED, Salt Lake City, UT) to drive protons into the skin. This constituted one trial, and different experimental areas of skin were used for each trial. Saline $(0.9 \% \mathrm{NaCl} \mathrm{w} / \mathrm{v})$ was used in the iontophoresis chamber as a control.

Magnitude of perceived pain rating. The magnitude of the perceived pain was assessed continually throughout the $4 \mathrm{~min}$ trial using a visual analog scale (VAS). Briefly, a scale was viewed on the monitor of a computer with the ends labeled " 0 " and " 100 ." Subjects were told that 0 represented no pain and 100 represented the worst pain imaginable. The volunteers were then prompted every $20 \mathrm{sec}$ by the computer to rate the pain they were experiencing at the experimental site, and they indicated the level of perceived pain by moving a pointer on the VAS scale with a computer mouse.

Modulation of acid pain by heat. To determine whether heating the experimental skin area augmented acid-mediated pain, warmed and cooled solutions of low $\mathrm{pH}$ were perfused continuously through the iontophoretic chamber while the solutions were iontophoretically delivered to the skin. This was achieved by heating the solutions in a volumetric flask using a water bath. The flask was connected to the iontophoretic chamber via a gravity feed. The chamber was continuously drained via an outlet tube attached to the bottom of the chamber (i.e., at skin level) and continuously infused from an inlet tube on the opposite side of the chamber and above the outlet tube. Fluids flowed continuously through the chamber and over the skin. Hot or cold fluids were therefore in dynamic equilibrium with the skin. We measured the temperature at the skin surface (within the chamber) with a thermode held against the skin surface. The perfusion speed was such that the temperature in the flask and at the skin surface (measured by a digital thermometer; Hanna Instruments, Leighton Buzzard, UK) were very similar $\left( \pm 0.5^{\circ} \mathrm{C}\right)$.

Desensitization of the experimental skin by capsaicin. Some skin sites were treated repeatedly with topical capsaicin ( $1 \%$ in $20 \%$ ethanol, Tween 80 , and $80 \%$ saline solution, under an occlusion dressing for $2 \mathrm{hr}$ ) to desensitize the skin to capsaicin and to block TRPV1-mediated acid pain responses. Two hours later, a new capsaicin solution was applied to the same site. This was repeated so that subjects received five treatments on the first day and at least three treatments on the second day. The skin was deemed desensitized when no spontaneous burning pain was re- ported after capsaicin application, and experiments were performed $\sim 45$ min afterward.

Capsaicin-mediated hyperalgesia. To render the experimental skin hyperalgesic before iontophoresis of acid and saline, $1 \%$ capsaicin solution was applied to the skin and covered with an occlusion dressing for $45 \mathrm{~min}$. After such time, the spontaneous burning pain associated with capsaicin application had diminished, and an area of mechanical allodynia was confirmed by softly stroking the skin with a cotton wool swab.

Topical application of NSAID creams. Five hundred milligrams of commercially available topically applied NSAID creams, diclofenac (Voltarol gel 5\% w/v; Novartis, Horsham, UK) and ibuprofen (Nurofen gel 5\% w/v; Crookes Healthcare, Nottingham, UK) were applied to a $6 \times 4 \mathrm{~cm}^{2}$ area of skin and covered with an occlusion dressing for $1 \mathrm{hr}$ before iontophoresis of test solutions. All creams, NSAID and placebo (KY jelly, an inert lubricating jelly; Johnson and Johnson, Maidenhead, UK), were removed from the skin before iontophoresis using an alcohol swab.

Determination of the heat pain threshold. Skin pretreated with both diclofenac and ibuprofen was subjected to measured heating to determine whether either NSAID affected heat pain thresholds. A commercial thermal sensory analyzer (Medoc Advanced Medical Systems, Ramat Yishai, Israel) was used to heat the experimental skin area from a holding temperature of $32^{\circ} \mathrm{C}$ at $0.5^{\circ} \mathrm{C} / \mathrm{sec}$. Subjects were asked to indicate when the stimulus was clearly painful. This was repeated at least twice for each site, and the thresholds were averaged.

Subcutaneous injection of amiloride. One hundred microliters of amiloride solution (1 mM) were injected intradermally at two different sites on the volar surface of the forearms of volunteers. One hundred microliters of saline (Steripak, Cheshire, UK) were also intradermally injected into two other sites, and these were used as controls. Thirty minutes after the injections, acid or saline were iontophoresed into the treated experimental skin areas in a predetermined pseudorandom sequence.

Solutions. One milliliter of concentrated hydrochloric acid (Sigma, Poole, UK) was diluted in 11 of distilled water to make a $12 \mathrm{~mm}$ solution with a $\mathrm{pH}$ of approximately $\mathrm{pH} 2$. Sodium chloride (BDH Laboratory Supplies, Poole, UK) was dissolved in distilled water to make a $0.9 \% \mathrm{w} / \mathrm{v}$ saline solution. The capsaicin solution was made by dissolving $50 \mathrm{mg}$ of 8-methyl- $N$-vanillyl-6-nonenamide ( $99 \%$ capsaicin powder; Sigma) in $0.5 \mathrm{ml}$ of ethyl alcohol and $0.5 \mathrm{ml}$ of Tween 80 . Four milliliters of sterile saline (Steripak) were added to make the solution up to $1 \%$. Amiloride solution was made by dissolving amiloride hydrochloride (Sigma) in sterile saline to make a $1 \mathrm{~mm}$ solution.

Statistical analysis. Statistical analysis was performed using SigmaStat software. Tests for normality were performed on the data, and subsequently parametric (predominantly ANOVA) or nonparametric tests (Mann-Whitney rank sum test or ANOVA on ranks) were performed as appropriate. All data are expressed as mean \pm SEM, and a level of $5 \%$ was taken as significant.

\section{Results \\ Iontophoresis of acid induces pain in a dose-dependent manner}

Iontophoresis of acid induced a pricking pain that was perceived by all the subjects $(n=12)$. The onset of the perceived pain occurred within the first $20 \mathrm{sec}$ of iontophoresis and reached a peak after $\sim 1 \mathrm{~min}$ (Fig. $1 B$ ). During the subsequent $3 \mathrm{~min}$ of the iontophoretic trial, the perceived pain diminished (Fig. $1 B$ ). The acid-induced pain was significantly higher than that reported for saline for all currents $(0.1-0.4 \mathrm{~mA})(p<0.05$; Kruskal-Wallis ANOVA on ranks followed by Tukey test; $n=12$ subjects) showing that it was specific to the iontophoresis of acid and not caused by the current being used to deliver the solutions transdermally 


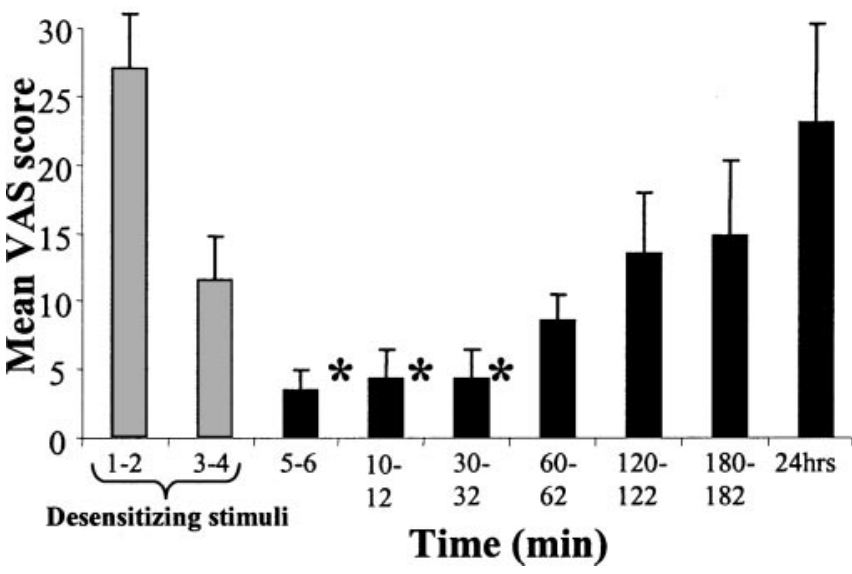

Figure 2. Time course of resensitization after desensitization with acid. Iontophoresis of acid $(0.3 \mathrm{~mA}, 4 \mathrm{~min})$ significantly desensitizes the skin to acid. The perceived acid pain is significantly reduced for $30 \mathrm{~min}$ after the desensitizing stimuli. Recovery from desensitization takes much longer than the initial desensitizing stimuli and does not fully recover until $24 \mathrm{hr}$ later. Asterisk denotes a significant difference $(p<0.05)$.

(Fig. $1 A, C$ ). Increasing the current from 0.1 to $0.4 \mathrm{~mA}$ significantly increased the magnitude of the perceived pain $(p<0.05$; Kruskal-Wallis ANOVA on ranks followed by Tukey test; $n=12$ subjects) except for the lowest currents used, showing that the acid-mediated pain increases in a dose-dependent manner (Fig. $1 A)$.

\section{Desensitization of acid pain and time course of recovery} Experiments presented above (Fig. $1 B$ ) showed that acid pain desensitizes fairly rapidly. To assess the time course of recovery, we iontophoresed acid into normal skin for $4 \mathrm{~min}$ as a desensitizing stimulus and then tested the same skin area at different time points after the desensitizing stimulus. We found (as before) that the mean VAS score was highest within the first minute and declined rapidly during the subsequent 3 min of the desensitizing stimulus (Fig. 2). The mean VAS score was lowest in the 2 min time period immediately after the desensitizing stimulus and was significantly different from the mean VAS score obtained from the first 2 min of the iontophoretic trial $(p<0.05$; KruskalWallis ANOVA on ranks followed by Dunn's test; $n=6$ ). The mean VAS scores obtained at 10-12 min and 30-32 min after the desensitizing stimuli were also statistically lower than the mean VAS score recorded during the first $2 \mathrm{~min}$ of the desensitizing stimuli ( $p<0.05$; Kruskal-Wallis ANOVA on ranks followed by Dunn's test; $n=8$ and $n=5$, respectively). At subsequent time points after the desensitizing stimulus, the mean VAS score gradually recovered until it reached the preconditioning value after 24 hr (Fig. 2).

\section{Capsaicin-mediated hyperalgesia potentiates acid pain}

Application of a 1\% capsaicin solution acutely sensitizes the skin and induces a spontaneous burning pain. The capsaicin-induced pain disappears after $\sim 45 \mathrm{~min}$ with this form of application but leaves the skin hyperalgesic for an additional 1-2 hr. After treatment with capsaicin and after the spontaneous pain had disappeared, we iontophoresed acid and saline into the treated skin sites, and, as a control, also into normal skin on the other forearm. When acid was iontophoresed into the hyperalgesic skin, the subjects' average pain rating was increased by approximately one-third, when compared with the mean VAS score obtained when acid was iontophoresed into normal skin (Fig. 3A). This

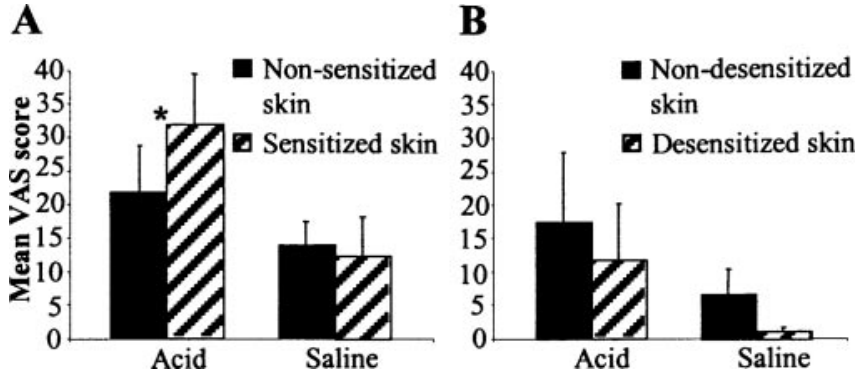

Figure 3. Mean VAS scores experienced when acid and saline were iontophoresed into skin acutely sensitized and desensitized with $1 \%$ capsaicin. $A$, Sensitizing the experimental skin with capsaicin significantly increases the perceived acid-mediated pain when compared with nonsensitized skin. Sensitizing the skin with capsaicin does not increase the pain experienced when saline is iontophoresed, indicating the sensitizing phenomena is specific for acid pain ( $n=12)$. $B$, Desensitizing the experimental skin with capsaicin does not significantly alter the perceived acid-mediated pain when desensitized and nondesensitized skin are compared $(n=5)$. Asterisk denotes a significant difference ( $p<0.05$ ).

increase in average pain score was significant when analyzed statistically $(p<0.05$; Kruskal-Wallis ANOVA on ranks followed by Tukey test; $n=12$ ). When saline was iontophoresed, there was no difference between normal and hyperalgesic skin $(p<0.05$; Kruskal-Wallis ANOVA on ranks followed by Tukey test; $n=12$ ) (Fig. $3 A$ ), and therefore the increase in perceived pain was specific to the acid stimuli. The sensitization of acid detection caused by capsaicin could arise because capsaicin-sensitive afferents participate in the signaling of the acid-mediated pain, but it could also arise from a more generalized sensitization in which the activation of TRPV1-expressing sensory terminals after the application of capsaicin modulates the properties of other sensory modalities. An example of the latter is the mechanical allodynia seen after application of capsaicin (LaMotte et al., 1991).

\section{Effect on acid-mediated pain of desensitizing skin} with capsaicin

Repeated application of $1 \%$ capsaicin solution desensitizes skin after approximately five to eight applications, so that the capsaicin application is no longer painful. After desensitizing the skin in this way, the average VAS score for iontophoresed acid (0.3 $\mathrm{mA}$ ) was reduced somewhat (Fig. 3B). However, this change was not significantly different from the mean VAS score obtained when acid is iontophoresed into normal nondesensitized skin ( $p>0.05$; Kruskal-Wallis ANOVA on ranks followed by Tukey test; $n=5)$. Similarly, the mean VAS score for saline iontophoresis is also decreased in desensitized skin when compared with normal skin (Fig. $3 B$ ), and again the reduction of the VAS score was not significantly different $(p>0.05$; Kruskal-Wallis ANOVA on ranks followed by Tukey test; $n=5$ ). This lack of a significant reduction in the acid-induced pain strongly suggests that the acid detection mechanism is not TRPV1 receptors, because these had been completely desensitized by the repeated application of capsaicin.

\section{Topical NSAIDs decrease acid-mediated pain}

Previous topical application of diclofenac reduced VAS scores to iontophoresed acid ( $0.3 \mathrm{~mA})$ throughout the $4 \mathrm{~min}$ trial (Fig. 4). This reduction was statistically significant, when NSAID-treated and vehicle-treated skin mean VAS scores were compared $(p<$ 0.05; Kruskal-Wallis ANOVA on ranks followed by Tukey test; $n=15)$. The pain ratings for saline iontophoresis were not different when NSAID-treated and vehicle-treated skin were com- 


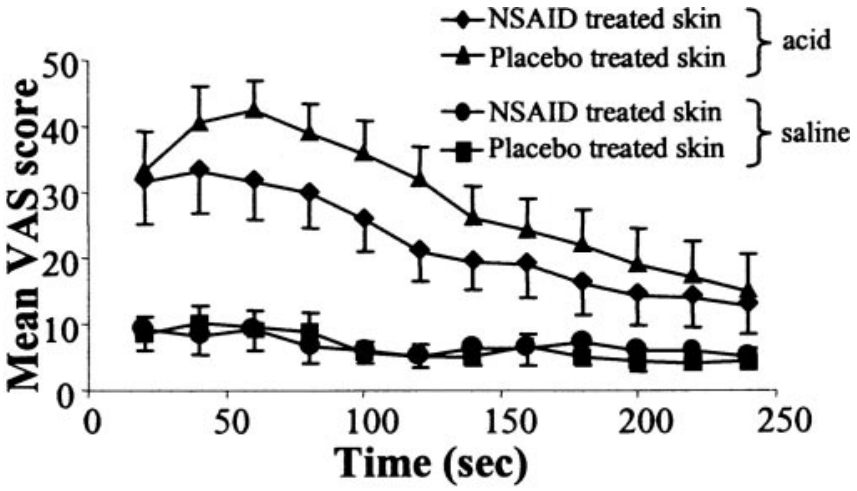

Figure 4. Time course of perceived pain during iontophoresis of acid on diclofenac-treated skin and placebo-treated skin. Except for the initial time point, topical application of diclofenac decreased the mean acid VAS score throughout the 4 min iontophoretic trial when compared with placebo-treated skin. Diclofenac does not affect the discomfort experienced during iontophoresis of saline $(n=15)$.
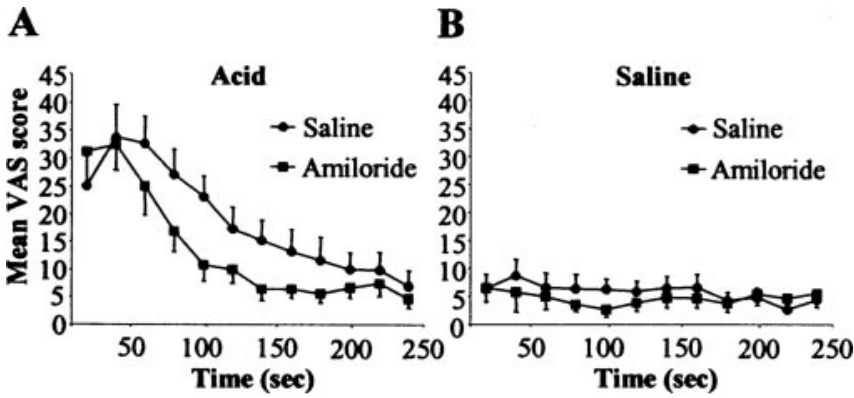

Figure 5. Time course of perceived pain during iontophoresis of acid and saline on amiloride-injected ( $1 \mathrm{~mm}$ ) and saline-injected skin. $A$, During the 4 min acid iontophoretic trial $(0.3 \mathrm{~mA})$, amiloride decreased the mean acid VAS scores at all time points except for the initial time point. $B$, During the iontophoresis of saline, amiloride marginally decreases the experienced pain associated with saline iontophoresis $(n=10)$.

pared ( $p>0.05$; Kruskal-Wallis ANOVA on ranks followed by Tukey test; $n=15)$, itself suggesting that the NSAID did not cause a nonspecific change in the sensitivity of nerve terminals or the conductivity of skin. To further test this hypothesis, we assessed the effects of topical NSAID on heat pain thresholds.

Diclofenac treatment did not significantly alter the heat pain thresholds compared with placebo control-treated skin $(p>$ $0.05 ; t$ test; $n=10$ ). This result also suggests that diclofenac has minimal effects on the functions of TRPV1.

The effects of topical ibuprofen were similar to those of diclofenac, i.e., previous topical application of ibuprofen reduced VAS scores to acid iontophoresis. However, after ibuprofen, the inhibition was only clear during the first $2 \mathrm{~min}$ of acid iontophoresis. As a result, the degree of inhibition of acid pain by ibuprofen, averaged over the entire period of acid application, was only $\sim 50 \%$ of that seen with diclofenac (Fig. 4 ).

\section{Amiloride inhibits acid-mediated pain}

To investigate the effect of amiloride on acid-mediated pain, 100 $\mu \mathrm{l}$ of a $1 \mathrm{~mm}$ solution of amiloride hydrochloride (Sigma) was injected subcutaneously into the volar surface of the forearm. Except for the initial time point, subcutaneous injection of amiloride reduced VAS scores to iontophoresed acid $(0.3 \mathrm{~mA})$ throughout the $4 \mathrm{~min}$ iontophoretic trial (Fig. 5A). This reduction was statistically significant, when amiloride-injected and saline-injected experimental skin mean VAS scores were com-

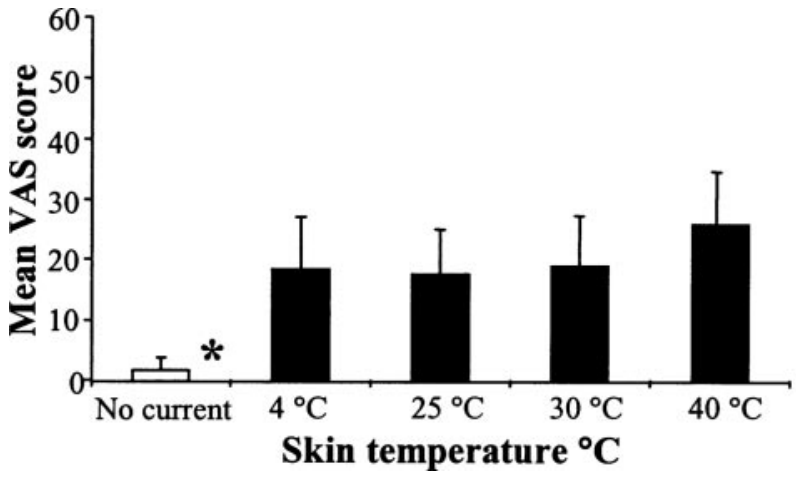

Figure 6. Mean VAS scores experienced during simultaneous iontophoresis of acid (0.3 mA) and heating of the experimental skin. Heating the skin to subthreshold heat pain temperatures does not significantly potentiate the experienced acid pain $(n=8)$. Asterisk denotes a significant difference $(p<0.05)$

pared ( $p<0.05$; Kruskal-Wallis ANOVA on ranks followed by Tukey test; $n=10)$. In contrast, subcutaneous injection of amiloride had no effect on the average saline pain score $(p>0.05$; Kruskal-Wallis ANOVA on ranks followed by Tukey test; $n=10$ ) (Fig. $5 B$ ), indicating that the inhibitory effects of amiloride are specific for acid-induced pain.

\section{Effects of heating on acid-mediated pain}

The effect of temperature on acid-induced pain was analyzed at different skin temperatures. The temperature of the experimental skin was changed by either heating or cooling the acid solution. As shown in Figure 6, there were only modest changes in acidinduced pain at different skin temperatures. Only the comparison between 40 and $25^{\circ} \mathrm{C}$ differed significantly $(p<0.05$; Kruskal-Wallis ANOVA with post hoc analysis by the Tukey test; $n=8$ ) with the VAS increased by a factor of 1.4 between 30 and $40^{\circ} \mathrm{C}$.

\section{Discussion}

Previous research has identified a number of ion channels, the properties of which suggest that they might be involved in signaling acidosis. In this study we have used experimental iontophoresis to deliver acid into the skin of human volunteers to investigate the properties of acid-induced pain in humans, with the aim of examining the contribution of the main candidate ion channels.

\section{Acid-induced pain}

In the majority of subjects, iontophoresis of acid caused moderate pain. This was dose-dependent for the currents used (0.1-0.4 $\mathrm{mA}$ ) and reached a peak after $\sim 1$ min of iontophoresis. Additional iontophoresis of acid resulted in progressive desensitization. This contrasts with results of previous experiments from other laboratories that report acid causes prolonged pain throughout the presentation of the acidic stimulus (Steen et al., 1995b). However, this difference in the time courses of the acid pain could be attributable to the different methods used to deliver acid subcutaneously. We used iontophoresis of acidic solutions, whereas the previous experiments used continuous intradermal perfusion. It is possible that these two different delivery regimes deliver acid at different $\mathrm{pHs}$ to the cutaneous nociceptor terminals, which could affect the rate of desensitization. Also in the previous studies, infusion of acidic buffers was such that average VAS scores of $20 \%$ were typically obtained, whereas in our experiments the most prominent desensitization was associated with higher VAS scores. However, it is also possible that the ionto- 
phoresis of protons and the subsequent vasodilation of the experimental skin could enhance the removal of protons and significantly affect the time constant of desensitization. In our experiments, recovery from desensitization took many hours (Fig. 2). It is not clear what underlies this prolonged desensitization. Acid-induced currents recorded from sensory neurons in vitro do show prominent desensitization, but this typically occurs over milliseconds or seconds, and recovery in seconds or minutes. One possible explanation is that we have delivered acid to the peripheral terminals of nociceptors, whereas electrophysiological measurements deliver acid to neuronal cell bodies. It is interesting that psychophysical responses to ATP iontophoresis in humans also show prolonged desensitization with similar discrepancies with electrophysiological data (Hamilton et al., 2000). We do know that desensitization in our experiments is not a result of the iontophoretic technique because previous iontophoresis of saline does not significantly reduce responses to protons (our unpublished data).

\section{Identity of the acid sensor}

We investigated the possible identity of the acid-sensing mechanism by comparing results obtained in the human psychophysical experiments presented in this paper with the known properties of ion channels whose activation by acid has been well documented: ASICs and TRPV1.

Effect of activation and desensitization of TRPV1 on $p H$ responses Acutely sensitizing skin with capsaicin significantly enhanced the perceived acid pain (Fig. 3A). This result is consistent with TRPV1 being the acid sensor. However, capsaicin injection sensitizes the skin to a wide range of stimuli, including non-noxious thermal and mechanical stimuli (LaMotte et al., 1991), and it is possible that the sensitization to acid is a reflection of the development of peripheral and/or central sensitization and does not necessarily reflect sensitization of the acid sensor itself. Conversely, chronically desensitizing skin with capsaicin did not significantly reduce acid pain (Fig. $3 B$ ). Because this treatment had completely desensitized TRPV1 receptors, as shown by the lack of any response to capsaicin, the observation that acid detection is unaffected strongly implies that ion channels other than TRPV1 contribute to physiological acid sensation.

\section{Effect of inhibitors of ASICs: NSAIDs}

To investigate a possible role of ASICs in signaling acid pain and to elucidate which ASIC subtypes may be involved, we investigated the effects of topically applied NSAIDs. NSAIDs decrease pain caused by inflammation (Tonussi and Ferreira, 1994), and a low $\mathrm{pH}$ environment around sites of inflammation has been proposed as a major contributing factor of inflammatory pain. In addition, more direct evidence that NSAIDs specifically inhibit acid pain has previously been demonstrated by Steen et al. (1995a). Growing evidence also suggests that inflammatory mediators can promote the expression of certain ASIC subtypes (Mamet et al., 2002, 2003). In addition to their ability to inhibit cyclo-oxygenase, NSAIDs may produce analgesia by other mechanisms. Voilley et al. (2001) showed that a number of NSAIDs inhibited homomeric ASIC subtypes in vitro. In particular, ibuprofen selectively inhibited ASIC1a, and diclofenac selectively inhibited ASIC3. Topical application of both ibuprofen and diclofenac was found to inhibit the initial acid-mediated pain in our studies (Fig. 4). In addition, diclofenac significantly reduced the acid-mediated pain throughout the iontophoretic trial, supporting a role for ASICs as a significant contributor to acid- induced pain. The reduction in acidotic pain was not attributable to a general analgesic action because neither ibuprofen nor diclofenac had any effect on the heat pain threshold. The specific inhibition of acidotic pain by NSAIDs thus supports a role for members of the ASIC family in generating acid pain.

\section{Effect of inhibitors of ASICs: amiloride}

The majority of ASIC channels expressed in vitro are inhibited by amiloride (Waldmann et al., 1997a,b). Injection of amiloride was found to significantly attenuate the acid-mediated pain (Fig. 5), suggesting that amiloride-sensitive ASIC channels are responsible for a substantial proportion of the acid pain. These findings are consistent with previous observations that amiloride attenuates acid pain caused by bolus injections of acidic solutions (Ugawa et al., 2002), although of course it is unknown whether amiloride has other nonspecific actions in human skin.

\section{Responses to heat}

Heating the experimental skin to $40^{\circ} \mathrm{C}$ had only a small effect in potentiating the perceived acid pain (Fig. 6), in marked contrast to the pronounced effect of heat in activating TRPV1 (Caterina et al., 1997). The human psychophysical data therefore seems to indicate that TRPV1 does not play an important role in acid detection. There are two possible explanations why the acid response in humans do not seem to involve the activation of TRPV1 channels: either the sensory terminals involved in acid detection do not express TRPV1 or the acidification caused by iontophoresis of acid into human skin was less than that used in the in vitro experiments and was able to activate ASIC but insufficient to activate TRPV1. The first explanation seems unlikely in view of the widespread colocalization both of TRPV1 and ASIC expression in DRG neurons (Alvarez de la Rosa et al., 2002), and of physiological responses to acid and capsaicin (LaMotte et al., 1991; Steen et al., 1995b). The second explanation is supported by the observation that inward currents carried by ASICs can be activated by modest acidifications (Waldmann et al., 1999; Hesselager et al., 2004), whereas acidification to $\mathrm{pH} 6$ is required to achieve significant activation of TRPV1 (Tominaga et al., 1998).

\section{Candidate ion channels that signal acidosis}

It seems likely from the results presented above that members of the ASIC family are major contributors to the detection of tissue acidosis. The rapid inactivation of ASIC1 and 3, however, and their desensitization by even modest acidification, suggest that other combinations of ASICs or other ion channels are involved. Our results further suggest that TRPV1 plays a relatively minor role in signaling acid pain, at least in the conditions of our experiments, in which acidotic pain of moderate intensity was elicited. A disadvantage of using iontophoresis to deliver acid to the skin is that although the stimulus can be finely controlled, the $\mathrm{pH}$ attained within the skin cannot readily be measured. TRPV1 is modulated by acid at lower pHs than ASICs (Tominaga et al., 1998), and TRPV1 may play a more prominent role at extreme pHs.

In addition to ASICs and TRPV1, other ion channels could be involved in signaling acidosis. Speculatatively, both ATP receptors and G-protein-coupled inward rectifying potassium (GIRK) channels could also be involved in signaling acidosis. The ionotropic ATP receptor subunit $\mathrm{P} 2 \mathrm{X}_{2}$ and heteromeric $\mathrm{P} 2 \mathrm{X}_{2} / \mathrm{P} 2 \mathrm{X}_{3}$ receptors have been shown to be modulated by small decreases in pH (Stoop et al., 1997; Wildman et al., 1997), as has the metabotropic $\mathrm{P}_{2} \mathrm{Y}_{4}$ receptor (Wildman et al., 2003), whereas, the GIRK channels, which are involved in the analgesic effects of opioids 
(Ikeda et al., 2002), are also modulated by acidification. Extracellular acidosis augments certain GIRK currents, which would stabilize the resting membrane potential (Mao et al., 2002), and thus make neurons harder to excite. Intracellular acidosis inhibits certain GIRK channels (Mao et al., 2003), which could promote nociceptor excitation. Although our data suggest that ASICs play a significant role in the detection of acid pain, and therefore may represent novel pharmacological targets to be used in the prevention of acid pain, the involvement of other mechanisms cannot be excluded.

\section{References}

Alvarez de la Rosa D, Zhang P, Shao D, White F, Canessa CM (2002) Functional implications of the localization and activity of acid-sensitive channels in rat peripheral nervous system. Proc Natl Acad Sci USA 99:2326-2331.

Caterina MJ, Schumacher MA, Tominaga M, Rosen TA, Levine JD, Julius D (1997) The capsaicin receptor: a heat-activated ion channel in the pain pathway. Nature 389:816-824.

Chen CC, England S, Akopian AN, Wood JN (1998) A sensory neuron-specific, proton-gated ion channel. Proc Natl Acad Sci USA 95:10240-10245.

Garber K (2003) Why it hurts: researchers seek mechanisms of cancer pain. J Nat Cancer Inst 95:770-772.

Gründer S, Geisler HS, Bässler EL, Ruppersberg JP (2000) A new member of acid-sensing ion channels from pituitary gland. NeuroReport 11:1607-1611.

Hamilton SG, Warburton J, Bhattacharjee A, Ward J, McMahon SB (2000) ATP in human skin elicits a dose-related pain response which is potentiated under conditions of hyperalgesia. Brain 123:1238-1246.

Hesselager M, Timmermann DB, Ahring PK (2004) pH dependency and desensitization kinetics of heterologously expressed combinations of acid-sensing ion channel subunits. J Biol Chem 279:11006-11015.

Ikeda K, Kobayashi T, Kumanishi T, Yano R, Sora I, Niki H (2002) Molecular mechanisms of analgesia induced by opioids and ethanol: is the GIRK channel one of the keys? Neurosci Res 44:21-31.

Issberner U, Reeh PW, Steen KH (1996) Pain due to tissue acidosis: a mechanism for inflammatory and ischemic myalgia? Neurosci Lett 208:191-194.

Kellenberger S, Schild L (2002) Epithelial sodium channel/degenerin family of ion channels: a variety of functions for a shared structure. Physiol Rev 82:735-767.

Krishtal OA, Pidoplichko VI (1981) Receptor for protons in the membrane of sensory neurons. Brain Res 214:150-154.

LaMotte RH, Shain CN, Simone DA, Tsai EF (1991) Neurogenic hyperalgesia: psychophysical studies of underlying mechanisms. J Neurophysiol 66:190-211.

Lingueglia E, de Weille JR, Bassilana F, Heurteaux C, Sakai H, Waldmann R, Lazdunski M (1997) A modulatory subunit of acid sensing ion channels in brain and dorsal root ganglion cells. J Biol Chem 272:29778-29783.

Mamet J, Baron A, Lazdunski M, Voilley N (2002) Proinflammatory mediators, stimulators of sensory neuron excitability via the expression of acid-sensing ion channels. J Neurosci 22:10662-10670.

Mamet J, Lazdunski M, Voilley N (2003) How nerve growth factor drives physiological and inflammatory expressions of acid-sensing ion channel 3 in sensory neurons. J Biol Chem 278:48907-48913.

Mao J, Li L, McManus M, Wu J, Cui N, Jiang C (2002) Molecular determinants for activation of G-protein-coupled inward rectifier $\mathrm{K}^{+}$(GIRK) channels by extracellular acidosis. J Biol Chem 277:46166-46171.

Mao J, Wu J, Chen F, Wang X, Jiang C (2003) Inhibition of G-protein- coupled inward rectifying $\mathrm{K}^{+}$channels by intracellular acidosis. J Biol Chem 278:7091-7098.

Olson TH, Riedl MS, Vulchanova L, Ortiz-Gonzalez XR, Elde R (1998) An acid sensing ion channel (ASIC) localises to small primary afferent neurons in rat. NeuroReport 9:1109-1113.

Pan HL, Longhurst JC, Eisenach JC, Chen SR (1999) Role of protons in activation of cardiac sympathetic C-fibre afferents during ischaemia in cats. J Physiol (Lond) 518:857-866.

Price MP, Snyder PM, Welsh MJ (1996) Cloning and expression of a novel human brain $\mathrm{Na}+$ channel. J Biol Chem 271:7879-7882.

Steen KH, Reeh PW (1993) Sustained graded pain and hyperalgesia from harmless experimental tissue acidosis in human skin. Neurosci Lett 154:113-116.

Steen KH, Reeh PW, Kreysel HW (1995a) Topical acetylsalicylic, salicylic acid and indomethacin suppress pain from experimental tissue acidosis in human skin. Pain 62:339-347.

Steen KH, Issberner U, Reeh PW (1995b) Pain due to experimental acidosis in human skin: evidence for non-adapting nociceptor excitation. Neurosci Lett 199:29-32.

Stoop R, Surprenant A, North RA (1997) Different sensitivities to pH of ATP-induced currents at four cloned P2X receptors. J Neurophysiol 78:1837-1840.

Stubbs M, McSheehy PM, Griffiths JR, Bashford CL (2000) Causes and consequences of tumour acidity and implications for treatment. Mol Med Today 6:15-19.

Sutherland SP, Benson CJ, Adelman JP, McCleskey EW (2001) Acidsensing ion channel 3 matches the acid-gated current in cardiac ischemiasensing neurons. Proc Natl Acad Sci USA 98:711-716.

Tominaga M, Caterina MJ, Malmberg AB, Rosen TA, Gilbert H, Skinner K, Raumann BE, Basbaum AI, Julius D (1998) The cloned capsaicin receptor integrates multiple pain-producing stimuli. Neuron 21:531-543.

Tonussi CR, Ferreira SH (1994) Mechanism of diclofenac analgesia: direct blockade of inflammatory sensitization. Eur J Pharmacol 251:173-179.

Ugawa S, Ueda T, Ishida Y, Nishigaki M, Shibata Y, Shimada S (2002) Amiloride-blockable acid-sensing ion channels are leading acid sensors expressed in human nociceptors. J Clin Invest 110:1185-1190.

Voilley N, de Weille J, Mamet J, Lazdunski M (2001) Nonsteroidal antiinflammatory drugs inhibit both the activity and the inflammationinduced expression of acid-sensing ion channels in nociceptors. J Neurosci 21:8026-8033.

Waldmann R, Champigny G, Bassilana F, Heurteaux C, Lazdunski M (1997a) A proton-gated cation channel involved in acid-sensing. Nature 386:173-177.

Waldmann R, Bassilana F, de Weille J, Champigny G, Heurteaux C, Lazdunski M (1997b) Molecular cloning of a non-inactivating proton-gated $\mathrm{Na}^{+}$channel specific for sensory neurons. J Biol Chem 272:20975-20978.

Waldmann R, Champigny G, Lingueglia E, De Weille JR, Heurteaux C, Lazdunski M (1999) $\mathrm{H}(+)$-gated cation channels. Ann NY Acad Sci 868:67-76.

Wildman SS, King BF, Burnstock G (1997) Potentiation of ATP-responses at a recombinant $\mathrm{P} 2 \times 2$ receptor by neurotransmitters and related substances. Br J Pharmacol 120:221-224.

Wildman SS, Unwin RJ, King BF (2003) Extended pharmacological profiles of rat P2Y2 and rat P2Y4 receptors and their sensitivity to extracellular $\mathrm{H}^{+}$and $\mathrm{Zn}^{2+}$ ions. Br J Pharmacol 140:1177-1186.

Xie J, Price MP, Berger AL, Welsh MJ (2002) DRASIC contributes to $\mathrm{pH}$ gated currents in large dorsal root ganglion sensory neurons by forming heteromultimeric channels. J Neurophysiol 87:2835-2843. 